Background The purpose of this study was to identify trends in survival and chemotherapy use, during the duration of the National Lung Cancer Audit (NLCA), for individuals with smallcell lung cancer (SCLC) in England.

Methods We used data from the NLCA database to identify people with histologically proven SCLC from 2004 to 2009. We used Hospital Episode Statistics (HES) database and NLCA to identify patients with codes of receiving chemotherapy, while NLCA was used to identify radiotherapy. We calculated the median survival by stage and observed the changing patient features of the cohort. We also looked at the proportions of patients with records of chemotherapy and/or radiotherapy over the years.

Results 11,603 patients were diagnosed with SCLC in our cohort. The median survival was 6 months; 1 year for limited stage and 4 months for extensive stage. $70 \%$ received chemotherapy and this proportion did not change overtime. Patient features including sex, age and performance status remained stable throughout the years $\left(\chi^{2}\right.$ p-value $0.25,0.93 \& 0.08$ respectively). There has been an increase in the proportion of patients that had a record of receiving chemotherapy and radiotherapy each year in both limited and extensive stage disease (from 19\% to $36 \%$ in limited \& from $10 \%$ to $17 \%$ in extensive stage from 2004 to 2009) (table 1). Patients who had a record for chemotherapy and radiotherapy had better survival in days compared with those who received only chemotherapy or no treatment independent of stage (334 days for chemotherapy \& radiotherapy vs. 240 days for chemotherapy alone, 82 days for radiotherapy alone $\& 25$ days for no treatment).

Conclusion Since 2004, when the NLCA was established, the recorded use of chemotherapy and survival in days has remained static. We have found an increasing trend in patients receiving chemotherapy \& radiotherapy which corresponds to better survival compared with other treatments. We were unable to see if these patients received these therapies for palliative or curative purpose; however the new chemotherapy and radiotherapy database soon to be linked with the NLCA would be better suitable to answer these questions.

\section{P15 IDENTIFYING PATIENTS WHO RECEIVE CHEMOTHERAPY FOR SMALL-CELL LUNG CANCER USING LARGE DATASETS}

${ }^{1} \mathrm{HA}$ Powell, ${ }^{1} \mathrm{U}$ Tata, ${ }^{2}$ RA Stanley, ${ }^{3} \mathrm{DR}$ Baldwin, ${ }^{1} \mathrm{RB}$ Hubbard; ${ }^{1}$ University of Nottingham, Nottingham, UK; ${ }^{2}$ Health and Social Care Information Centre, Leeds, UK; ${ }^{3}$ Nottingham University Hospitals NHS Trust, Nottingham, UK

\subsection{6/thoraxjn-2013-204457.165}

Introduction The National Lung Cancer Audit (NLCA) has collected data on primary lung cancer in England since 2004, and has now been linked with Hospital Episodes Statistics (HES) for research into inequalities in access to treatment. How well these two large datasets capture chemotherapy for small cell lung cancer (SCLC) is not known.

Methods We identified all cases of SCLC in the NLCA diagnosed between January 2004 and March 2012. We calculated the proportion of patients with a HES code for chemotherapy, and the proportion with a start date for chemotherapy in the NLCA, within 6 months of diagnosis. We inspected survival curves for people with a chemotherapy record in HES only or the NLCA only, people who had records of chemotherapy in both databases (who we could be reasonably sure had chemotherapy), and those with no record of chemotherapy. We assessed whether the results changed over time as case ascertainment in the NLCA increased from $19 \%$ to $98 \%$ between 2004 and 2009 .

Results We identified 18,398 cases of histologically confirmed SCLC; 9,484 (52\%) had chemotherapy records in both databases and 5,100 (28\%) had no record of chemotherapy in either. 737 patients (4\%) had chemotherapy recorded only in HES and 2,539 $(14 \%)$ only in the NLCA. For people with a record of chemotherapy in a single database (NLCA only or HES only) survival was similar to that of people with records of chemotherapy in both datasets (figure 1); the average age, stage and performance status was also very similar for people in these three groups. Survival patterns were the same when we analysed the data by year of diagnosis however the proportions with chemotherapy records in HES only or the NLCA only decreased to $3 \%$ and $12 \%$ respectively in 2011 .

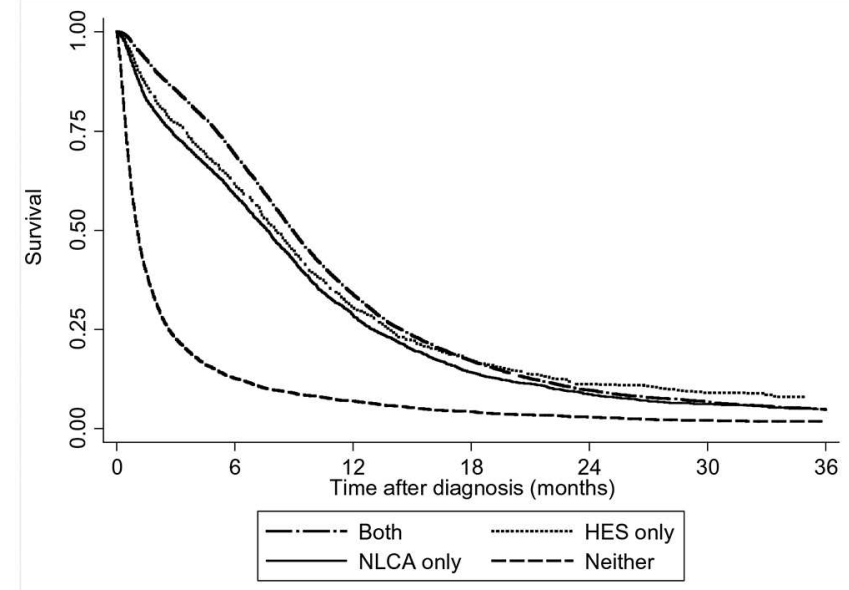

Abstract P15 Figure 1. Survival after diagnosis for people with SCLC according to records of chemotherapy

Conclusion Our results suggest that it is best to identify people who received chemotherapy using data in the NLCA and HES combined. A record of chemotherapy in either database appears to be a valid means of determining who received chemotherapy but if a single database is used the proportion treated is likely to be an under-estimate.

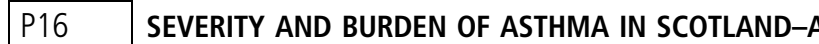 FULL POPULATION STUDY USING THE PRESCRIBING INFORMATION SYSTEM}

${ }^{1} \mathrm{MFC}$ Steiner, ${ }^{1} \mathrm{G}$ Devereux, 'S Turner, 'J McLay, ${ }^{2} \mathrm{~B}$ Bishop, ${ }^{2} \mathrm{G}$ Wyper; ${ }^{1}$ University of Aberdeen, Aberdeen, United Kingdom; ${ }^{2}$ NHS National Services Scotland, Edinburgh, United Kingdom

\subsection{6/thoraxjnl-2013-204457.166}

In contrast to many epidemiological studies of asthma that have been limited to samples assumed to be representative of the national population from which they are drawn we have used routinely collected drug dispensing data for the whole Scottish population. Our aim was to use NHS Scotland's Prescribing Information System (PIS) to describe asthma prevalence, asthma severity (BTS treatment steps) and asthma control (exacerbations, hospital / A\&E episodes) in children and young adults. Methods For $>95 \%$ of dispensed prescriptions in primary care between December 2009 and December 2012 a valid patient identifier is available including some socio-demographical characteristics (age-group, sex, socioeconomic status). Data were also linked to hospital admission and A\&E event data to identify 
exacerbations and exclusion criteria. The analysis was limited to patients aged $=45$ years to reduce contamination by COPD. Patients with non-asthma respiratory diagnoses were excluded. Results In the 3 year period about 395,000 patients received repeated medications for asthma with a monthly point prevalence of 255,000 asthma patients; equating to a prevalence of $7.0 \%$ of the 3.6 million people aged $0-45$ registered with a GP in Scotland. These patients collected 5.6 million prescriptions for inhaled/oral asthma therapies and 253,000 short courses of oral corticosteroids were dispensed to these patients in primary care. Patients were categorised on a daily basis into BTS Steps 15 according their medication use. As of June 2011: 83,663 (32.4\%) of patients were in Step 1;99,374 (38.5\%) were in Step 2; 42,555, (16.5\%) were Step 3; 27852 (10.8\%) were Step 4 , and 4,500 patients $(1.7 \%)$ were Step 5 receiving daily oral corticosteroids. Table 1 presents the exacerbation rates for these patients, $1.9 \%$ of the patients received an emergency short course of prednisolone,. Conclusion This current and whole population database indicates that in Scotland asthma prevalence is about $7 \%$ in this age group. The proportion of asthma patients treated at Steps 3-5 (25\%) is greater than the often quoted $15 \%$. Although proportionately small, a sizeable number of patients $(\sim 7000)$ with Step 1 and 2 asthma had at least one exacerbation requiring short course prednisolone and/or hospital contact over a three year period.

$\begin{aligned} & \text { Abstract P16 Table 1. Dispensed inhaled therapy and A\&E } \\
& \text { attendance data from the Scottish NHS databases }\end{aligned}$
\begin{tabular}{llllll}
\hline BTS Steps & $\mathbf{1}$ & $\mathbf{2}$ & $\mathbf{3}$ & $\mathbf{4}$ & $\mathbf{5}$ \\
\hline $\mathrm{N}$ & 83,663 & 99,374 & 42,555 & 27,852 & 4,500 \\
$\% \geq 1$ short-course prednisolone & $1.0 \%$ & $2.0 \%$ & $2.2 \%$ & $4.3 \%$ & $0.5 \%$ \\
$\% \geq 1$ A\&E attendance with asthma over 3 years & $1.4 \%$ & $2.7 \%$ & $3.3 \%$ & $4.5 \%$ & $10.9 \%$ \\
\hline
\end{tabular}

\section{P17 THE BURDEN OF ICS/LABA-TREATED ASTHMA PATIENTS IN THE UK ADULT POPULATION}

${ }^{1} \mathrm{D}$ Price, ${ }^{2} \mathrm{~N}$ Mathieson, ${ }^{2} \mathrm{~A}$ Mulgirigama, ${ }^{2} \mathrm{~A}$ Scowcroft, ${ }^{3} \mathrm{R}$ Pedersini, ${ }^{3} \mathrm{G}$ Isherwood, ${ }^{4}$ ID Pavord; ' $A$ Academic Centre of Primary Care, University of Aberdeen, Aberdeen, UK; ${ }^{2}$ Boehringer Ingelheim Ltd UK, Berkshire, UK; ${ }^{3}$ Kantar Health, Epsom, UK; ${ }^{4}$ University Hospitals of Leicester NHS Trust, Institute for Lung Health, Glenfield Hospital, Leicester, UK

\subsection{6/thoraxjn-2013-204457.167}

Objectives According to NHS QOF (Quality and Outcomes Framework) figures, 3.3 million UK citizens have asthma. Previous studies have shown an association of asthma with increased direct and indirect healthcare costs, but similar studies have not been conducted specifically for UK asthma patients. The aim of the current study is to assess the impact of poor asthma control on UK patients treated with ICS + LABA maintenance treatment.

Methods Data were from the 2010 and 2011 UK National Health and Wellness Survey (NHWS), an Internet-based questionnaire from a representative sample of UK adults stratified by age and gender. 701 respondents self-reported a diagnosis of asthma without concomitant COPD, chronic bronchitis, or emphysema and were currently being treated with ICS + LABA.

Patients Not Well Controlled (NWC) according to ACT (score $<20$ ) were compared to well-controlled (WC) patients (score $\geq 20$ ) on demographics, medications, health status, BMI, comorbidities, adherence (MMAS-4), healthcare use (number of physician visits, emergency visits and hospitalizations), work productivity and activity impairment (WPAI) and health-related quality of life (HR-QoL) (SF-12).

Results A greater proportion of the 452 NWC patients (64\% of the overall sample) go to emergency $(21 \%$ vs. $14 \%, \mathrm{p}=0.016)$ or are hospitalised $(13 \%$ vs. $8 \%, \mathrm{p}=0.022)$, in comparison with the WC; Their mental and physical HR-QoL is lower (SF12 MCS: 43 vs. $47 / 100$; PCS: 40 vs. $48 / 100$; Health utility: 0.65 vs. $0.74 / 1.00$; all p's $<0.001$ ); while their work and activity impairment are greater: presenteeism $(23 \%$ vs. $11 \%, \mathrm{p}<$ $0.001)$, overall work impairment $(29 \%$ vs. $17 \%, \mathrm{p}<0.001)$ and activity impairment $(46 \%$ vs. $24 \%, \mathrm{p}<0.001)$. In the current sample, NWC did not show significantly different levels of adherence from WC $(50 \%$ vs. $55 \%, \mathrm{p}=0.361)$.

Conclusions Over 60\% UK ICS + LABA-treated adult patients are poorly controlled. Poor control is associated with lower HRQoL, greater healthcare use and productivity impairment, but not with significantly different levels of adherence to WC patients. The recognition of patients remaining symptomatic and utilising healthcare resource whilst treated with ICS + LABA maintenance therapy is an important step to improving their management.

\section{P18 IDIOPATHIC PULMONARY FIBROSIS SURVIVAL HAS NOT IMPROVED IN THE 21ST CENTURY; ANALYSIS OF CPRD GOLD PRIMARY CARE DATA}

${ }^{1}$ Tm Maher, ${ }^{2} \mathrm{H}$ Strongman, ${ }^{2} \mathrm{R}$ Boggon, ${ }^{3} \mathrm{I}$ Kausar; ${ }^{1}$ Royal Brompton Hospital, London, United Kingdom; ${ }^{2}$ Clinical Practice Research Datalink, London, United Kingdom; ${ }^{3}$ InterMune UK and Ireland, London, United Kingdom

\subsection{6/thoraxjnl-2013-204457.168}

Introduction and Objectives Idiopathic pulmonary fibrosis (IPF) is a progressive and invariably fatal disease. Historical cohort studies have reported a median survival of $2.8-3.2$ years $^{1}$. The UK has seen a number of important developments for IPF patients in the last year which it is hoped will translate to improved outcomes for individuals with this devastating disease. These include: the licensing and approval by NICE of the first anti fibrotic therapy, pirfenidone; specialist commissioning of ILD services; and publication of NICE IPF guidelines. If the impact of these important developments is to be measured it is vital that current IPF disease burden is understood.

Methods The incidence and mortality of IPF-Clinical Syndrome, using broad and narrow disease definitions, was assessed via the Clinical Practice Research Datalink (CPRD) GOLD dataset.

Results The incidence of broadly defined IPF-CS between 2000-2012 was 8.65 (95\% CI 8.40-8.90) per 100,000 person years and significantly increased over time (incidence rate ratio adjusted by gender, region and age category $=1.02$, $\mathrm{p}$ for linear trend $<0.0001$ ). Kaplan-Meier survival estimates show no significant survival difference by year of diagnosis (log rank test for equality of survivor functions, $\mathrm{p}=0.17$ ) with a median survival of 3.0 years (95\% CI 2.8-3.1). There was no significant survival difference for broad and narrow definitions (log rank test for equality of survivor functions, $\mathrm{p}=0.06$ ) validating the definition of IPF-CS.

Conclusions In keeping with previous data the incidence of IPFCS continues to rise and the survival time from diagnosis remains unchanged. The mortality burden of IPF-CS is therefore increasing. The results of this study provide an important benchmark against which the effects of changes in the management and delivery of care for individuals with IPF can be measured. 